\title{
Loop Filter Design for Phase-Locked Loops with Delay: A Multi-objective Control Approach
}

\author{
Y.S. Chou, Y.C. Chen, W.L. Mao and F.R. Chang
}

\begin{abstract}
Two loop filter design methods for phase-locked loops (PLLs) with loop delay are presented, which employs multi-objective control technique to deal with the various design objectives: small noise bandwidth and large gain and phase margins, etc. Trade-off among the conflicting objectives is made via recently developed convex optimization skill in conjunction with appropriate adjustment of certain design parameters. By applying first-order Padé approximation for the delay term, one of the design methods has the advantage that almost all except one of the filter poles can be assigned a priori. On the other hand, through applying a special coordinate transformation technique, a new PI form loop filter design is also presented. It is noted that both of the proposed methods are applicable to PLL of any order. Numerical simulation on nonlinear PLL model is performed which demonstrates the effectiveness of the proposed methods.
\end{abstract}

Index Terms-PLL, time delay, PI form filter, multi-objective control, linear matrix inequality (LMI).

\section{INTRODUCTION}

$\mathrm{S}_{\mathrm{b}}^{\mathrm{n}}$ NCE its invention, the PLL principle [1], [2], [3], [4] has been used in a wide spread of applications, such as carrier tracking [5], timing recovery [6], and servo control [7], [8], etc [9]. From the system's point of view, PLL is essentially a nonlinear system. The design of PLL with a sinusoidalcharacteristic phase detector using Lyapunov redesign technique can be found in [10], [11]. However, there is a difficulty in applying the proposed method to high-order loops. Besides, large decision delays frequently exist in modern receivers, which might degrade the performance of the systems, leading to smaller damping, larger settling time, reduction of the phase margin and even more causing to instability. Recently, a method based on linear model approximation was proposed which designs a loop filter that minimizes the phase error variance with guaranteed gain margin and phase margin in the presence of phase detector gain uncertainty and constant loop delay [12]. A complicated design procedure was provided via applying coprime

Y.S. Chou is with the Department of Electrical Engineering, Tamkang University, Taipei county, Taiwan (phone: +886-2-26215656 ext 3293; fax: +886-2-262 09814; e-mail: yung@ee.tku.edu.tw)

M. L. Mao is with the Department of Electronic Engineering, National Formosa University, Yulin county, Taiwan (e-mail: wlmao123@ yahoo.com.tw)

F.R. Chang and Y.C. Chen are with the Department of Electrical Engineering, National Taiwan University, Taipei, Taiwan. (e-mail: frchang @cc.ee.ntu.edu.tw,d95921008@ntu.edu.tw) factorization control theory [13] and quantitative feedback theory (QFT) [14]. In many cases, PI form filters which have all of the poles at the origin are favorable for some advantages [3] they brought in: infinite hold range and pull-in range (theoretically), and good phase tracking capability. However, the filters obtained by the approach just mentioned usually have poles not at the origin. Having noticed this point, an independent design procedure of PI form filter has also been addressed in [12] for second-order loop. Nevertheless, it can be checked that it is hard to extend the method therein to higher order loops. In [15] the same problem for PLLs with polytopic uncertainties is studied using another approach. Similarly, the filters obtained are generally not of PI form.

In this paper, two types of loop filters are considered: the general form filters (in which not all of the poles are locating at the origin) and the PI form filters. The design of the class of general form filters is transformed into multi-objective static state feedback synthesis problem, where the various design objectives: small noise bandwidth, good transient response [16], and large gain and phase margins [16] are all taken into consideration. Trade-off among them is made via linear matrix inequality (LMI) optimization [17], [18], [19], [20] in conjunction with appropriate adjustment of certain design parameters. Similarly, the synthesis of the class of PI form filters is formulated as a mixed $H_{2} / H_{\infty}$ control problem. A special coordinate transformation technique [21] is employed to deal with the problem. The paper is organized as follows. In Section II, the preliminaries and problem statement are given. In Section III, the design of the general form loop filters is presented. Section IV provides the PI form filter design. Section V shows the simulation results. Finally, Section VI gives the conclusion. The detailed definitions of the $\mathrm{H}_{2}$ and $H_{\infty}$ norm of stable transfer functions can be found in [22]. Throughout this paper, a signal in time domain and frequency domain are denoted by lower case and upper case, respectively.

\section{Preliminaries And Problem Statement}

\section{A. Basic model of PLL}

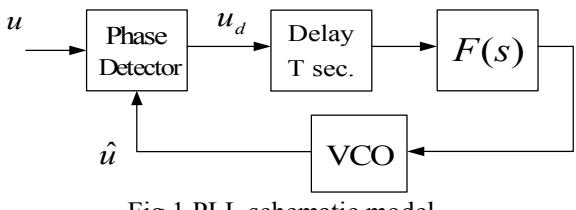

Fig.1 PLL schematic model 
The PLL model used here is depicted in Fig. 1, which consists of a phase detector, low-pass loop filter $F(s)$, and voltage controlled oscillator (VCO). The inputs to the phase detector are the two signals: the sum of the carrier and noise $n(t)$ that is stationary, Gaussian, bandpass and zero mean [4], i.e.,

$$
u(t)=\sqrt{2} A_{d} \sin \left(\omega_{0} t+w_{\theta}(t)\right)+n(t),
$$

and the VCO output

$$
\hat{u}(t)=\sqrt{2} \cos \left(\omega_{0} t+\hat{w}_{\theta}(t)\right) .
$$

The phase detector produces, assuming the high frequency term is eliminated by the low-pass filter, the output signal

$$
u_{d}(t)=A_{d}\left[\sin \left(w_{\theta}-\hat{w}_{\theta}\right)+n^{\prime}(t)\right]
$$

where $n^{\prime}$ represents the net effect caused by the noise $n$. For small phase errors and small input phase disturbance $w_{n}(t)$, the PLL can be further approximated by the linear model as depicted in Fig. 2.

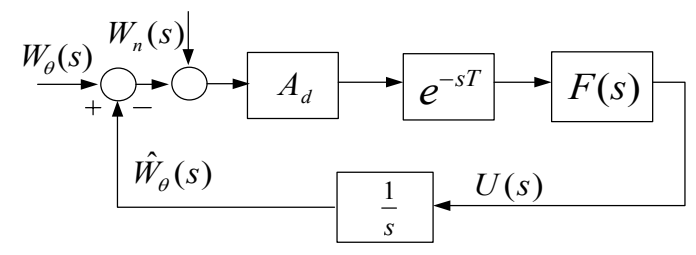

Fig. 2 PLL linear model

\section{B. The goal}

In view of Fig. 2, the goal of this paper is to design a filter $F(s)$ to achieve the following objectives:

(i) Closed-loop stability,

(ii) Perfect asymptotical tracking (i.e. $e(\infty)=0$ ) subject to the deterministic test signals $w_{\theta}(t)=t^{k}, \quad k=0,1,2, \ldots, m$ when $w_{n}=0$,

(iii) Good transient response (i.e., small settling time, small overshoot, etc),

(iv) Noise attenuation (assuming $w_{n}$ to be white noise),

(v) Large stability margin (i.e. gain margin (GM), phase margin (PM)).

Note that the variance of VCO output phase is given by

$$
\sigma_{\hat{w}_{\theta}}^{2}=\frac{1}{2 \pi} \int_{-\infty}^{\infty}\left|T_{\hat{w}_{\theta} w_{n}}(j \omega)\right|^{2} \Phi_{w_{n}}(\omega) d \omega
$$

where $T_{\hat{w}_{\theta} w_{n}}$ represents the transfer function from $w_{n}$ to $\hat{w}_{\theta}$, and $\Phi_{w_{n}}(\omega)$ is the power spectral density (PSD) function of the noise $w_{n}$. If $w_{n}$ is white Gaussian, i.e., $\Phi_{w_{n}}(\omega)^{+}=N_{0}$, then

$$
\sigma_{\hat{w}_{\theta}}^{2}=N_{0}\left\|T_{\hat{w}_{\theta} w_{n}}\right\|_{2}^{2}=2 N_{0} B_{n}
$$

where $B_{n}(\mathrm{~Hz})$ denotes the noise bandwidth of $T_{\hat{w}_{\theta} w_{n}}$. It is clear that small noise bandwidth $B_{n}$ leads to small variance of the VCO output phase. In terms of the loop filter design, this can be achieved by minimizing the $H_{2}$ norm of the closed-loop system $T_{\hat{w}_{\theta} w_{n}}$ over all the stabilizing filters.

On the other hand, the open-loop response should have enough gain and phase margins in order to guarantee good relative stability and well-behaved closed-loop response. It is mentioned in [12], [14] that these margins can be determined by the $H_{\infty}$ norm upper bounds of certain closed-loop system. Specifically, let $L(s)$ denote the open loop transfer function of Fig. 2. Assume a constant $\gamma$ or alternatively a constant $\delta$ satisfies the following $H_{\infty}$ norm condition:

(1)

$$
\left|\frac{L(j \omega)}{1+L(j \omega)}\right|<\gamma \quad\left(\text { resp. }\left|\frac{1}{1+L(j \omega)}\right|<\delta\right)
$$

A pair of lower bounds of the gain and phase margins is then determined by the formulas:

$$
20 \log \frac{\gamma+1}{\gamma} d B ; \quad 2 \sin ^{-1} \frac{1}{2 \gamma} \operatorname{deg}\left(\text { resp. } 20 \log \frac{\delta}{\delta-1} d B ; \quad 2 \sin ^{-1} \frac{1}{2 \delta} \operatorname{deg}\right)
$$

Note that condition (1) is equivalent to a $H_{\infty}$ norm bound constraint of the closed-loop system $T_{\hat{w}_{\theta} w_{\theta}}$. Thus minimizing the $H_{\infty}$ norm of the closed-loop system $T_{\hat{w}_{\theta} w_{\theta}}$ over all the stabilizing filters is expected to increase the gain and phase margins.

\section{Useful Analysis LMIs}

In the following, Lemma 1 [18] and Lemma 2 [18] present the $\mathrm{H}_{2}$ and $H_{\infty}$ norm constraints of a LTI system in terms of LMIs, respectively. Lemma 3 [19] and lemma 4 [19] state that all the eigenvalues of a square matrix lie in a prescribed region if and only if certain LMIs are feasible.

Lemma 1 [18]: Given a LTI system $H(s)=C(s I-A)^{-1} B$, and a positive value $v$. The following statements are equivalent.

(i) the LTI system $H(s)$ is stable and $\|H\|_{2}<v$.

(ii) there exist matrices $P=P^{T}$ and $Q=Q^{T}$ such that the following LMIs hold,

$$
\begin{gathered}
\left(\begin{array}{cc}
A P+P A^{T} & B \\
B^{T} & -I
\end{array}\right)<0,\left(\begin{array}{cc}
P & (C P)^{T} \\
C P & Q
\end{array}\right)>0, \\
\operatorname{Tr}(Q)<v^{2} .
\end{gathered}
$$

Lemma 2 [18]: Given a LTI system $H(s)=C(s I-A)^{-1} B+D$, and a positive value $\gamma$. The following statements are equivalent.

(i) The LTI system $H(s)$ is stable and $\|H\|_{\infty}<\gamma$.

(ii) There exists a matrix $P=P^{T}$ such that the following LMIs hold,

$$
\left(\begin{array}{ccc}
A P+P A^{T} & B & (C P)^{T} \\
B^{T} & -\gamma I & D^{T} \\
C P & D & -\gamma I
\end{array}\right)<0, \quad P>0 .
$$


Lemma 3 [19]: Given a square matrix $A$, all the eigenvalues of $A$ lie in the conic sector as shown in Fig. 3(a) if and only if there exists a matrix $X=X^{T}$ such that the following LMIs are satisfied,

$$
\begin{gathered}
\left(\begin{array}{cc}
\sin (\theta)\left(A X+X A^{T}\right) & \cos (\theta)\left(A X-X A^{T}\right) \\
\cos (\theta)\left(X A^{T}-A X\right) & \sin (\theta)\left(A X+X A^{T}\right)
\end{array}\right)<0, \\
X>0 .
\end{gathered}
$$

Lemma 4 [19]: Given a square matrix $A$, all the eigenvalues of $A$ lie in the vertical strip $\left(-h_{1},-h_{2}\right)$ as shown in Fig. 3(b) if and only if there exists a matrix $X=X^{T}$ such that the following LMIs are satisfied,

$$
\begin{gathered}
-2 h_{1} X-A X-X A^{T}<0, \\
2 h_{2} X+A X+X A^{T}<0, \\
X>0 .
\end{gathered}
$$

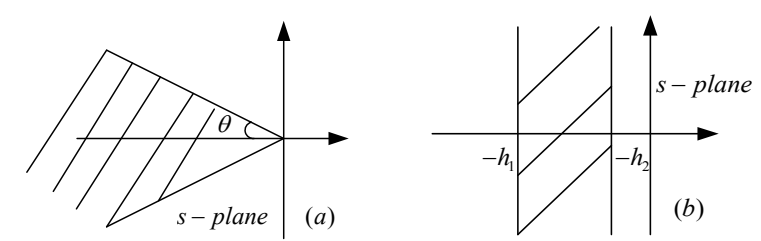

Fig.3 (a) conic sector (b) vertical strip $\left(-h_{1},-h_{2}\right)$

Note that for standard second-order systems and their approximations, placing the dominant poles in the conic sector region (see Fig. 3(a)) with smaller $\theta$ results in smaller percent overshoot [16]. Similarly, placing the dominant poles in the vertical strip (see Fig. 3(b)) with larger $h_{2}$ results in smaller settling time [16].

\section{General Form LoOp Filter Design}

In this section, a new method is proposed to design a loop filter for PLL. Firstly in part A, we generalize the technique in [23], [24] to transform the problem of designing a dynamic filter into a static state feedback synthesis problem. Secondly, in part B, a multi-objective state feedback synthesis technique is employed to find a loop filter to satisfy the design objectives mentioned in Section II.

\section{A. Problem reformulation}

To proceed, Fig. 2 is redrawn as Fig. 4 below

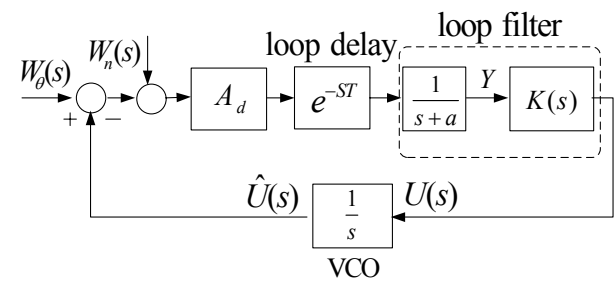

Fig. 4 PLL Linear model II

with loop filter $F(s)$ of the form

$$
F(s)=\frac{s}{s+a} \cdot \frac{1+(T / 2) \cdot s}{\left(1-f_{m+l+1}\right)+(T / 2) \cdot s} \cdot\left[\sum_{i=0}^{m} \frac{f_{i}}{s^{i}}+\sum_{i=m+1}^{m+l} \frac{f_{i}}{s-\beta_{i-m}}\right]
$$

where $a$ and $\beta_{j^{\prime} s}$ are the real poles of the filter which can be assigned a priori, $T$ denotes pure delay, and the real coefficients $f_{i^{\prime} s}$ are to be designed. Let $l=0$ denote the case that the component functions with poles at $\beta_{j^{\prime} s}$ are taken out from (3), i.e.,

$$
F(s)=\frac{s}{s+a} \cdot \frac{1+\frac{T}{2} s}{\left(1-f_{m+l+1}\right)+\frac{T}{2} s} \cdot\left[\sum_{i=0}^{m} \frac{f_{i}}{s^{i}}\right]
$$

In view of Fig. 4, the signal $Y(s)$ can be described by

$$
Y(s)=e^{-s T} \frac{A_{d}}{s+a}\left(W_{n}(s)+W_{\theta}(s)-\hat{U}(s)\right)
$$

Applying first-order Padé approximation for the delay term $e^{-s T}$ by

$$
e^{-s T} \approx \frac{1-T / 2 \cdot s}{1+T / 2 \cdot s}=: D(s)
$$

Then (4) becomes

$$
\begin{aligned}
s Y(s) \approx & -a Y(s)+A_{d} \hat{U}(s)-2 A_{d} \frac{1}{1+T / 2 \cdot s} \hat{U}(s) \\
& +\left[\begin{array}{ll}
A_{d} & A_{d}
\end{array}\right] \bar{D}(s)\left[\begin{array}{c}
W_{n}(s) \\
W_{\theta}(s)
\end{array}\right]
\end{aligned}
$$

where $\bar{D}(s)=\operatorname{diag}(D(s), D(s))$ is an all-pass transfer matrix. Define state vector $\bar{\xi}(s)$ in frequency domain as follows:

$\bar{\xi}(s):=\left[Y(s), \frac{1}{s} Y(s), \cdots, \frac{1}{s^{m}} Y(s), \frac{1}{s-\beta_{1}} Y(s), \cdots \frac{1}{s-\beta_{l}} Y(s), \frac{1}{1+T / 2 \cdot s} \hat{U}(s)\right]^{T}$

$=:\left[\xi_{1}(s), \xi_{2}(s), \xi_{3}(s), \cdots, \xi_{m+1+2}(s)\right]^{T}$.

Then (5) becomes

$s \xi_{1}(s)=-a \xi_{1}(s)-2 A_{d} \xi_{m+l+2}(s)+A_{d} \hat{U}(s)+\left[\begin{array}{ll}A_{d} & A_{d}\end{array}\right] \bar{D}(s)\left[\begin{array}{l}W_{n}(s) \\ W_{\theta}(s)\end{array}\right]$

Moreover, the other state equations read

$$
\begin{aligned}
& s \xi_{i}(s)=\xi_{i-1}(s), i=2,3, \cdots, m+1 \\
& s \xi_{i}(s)=\beta_{i-m-1} \cdot \xi_{i}(s)+\xi_{1}, i=m+2, \cdots, m+l+1 \\
& s \xi_{m+l+2}(s)=-2 / T \cdot \xi_{m+l+2}(s)+2 / T \cdot \hat{U}(s) .
\end{aligned}
$$

Set the observed output $Z(s):=\hat{U}(s)$ and $\bar{Y}(s):=\bar{\xi}(s)$. With the notation defined above it is easy to check that the dynamic filter of the prescribed form (3) is converted into a static state feedback in the new coordinate, i.e., $\hat{U}(s)=F_{s} \cdot \bar{Y}(s)$ where $F_{s}=\left[f_{0}, \cdots, f_{m+l+1}\right]$. Thus the original dynamic filter design problem is equivalently transformed to a static state feedback control problem as illustrated in Fig. 5

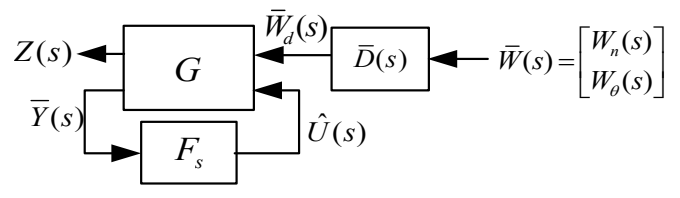

Fig. 5 The equivalent state feedback model 
with $G$ described by

$$
G\left\{\begin{array}{l}
s \bar{\xi}(s)=A \bar{\xi}(s)+B_{1} \bar{W}_{d}(s)+B_{2} \widehat{U}(s) \\
Z(s)=D_{12} \widehat{U}(s) \\
\bar{Y}(s)=\bar{\xi}(s)
\end{array}\right.
$$

where $\bar{W}_{d}(s)=\left[\begin{array}{ll}W_{d_{1}}(s) & W_{d_{2}}(s)\end{array}\right]^{T}:=\bar{D}(s) \cdot\left[\begin{array}{ll}W_{n}(s) & W_{\theta}(s)\end{array}\right]^{T}$,

$A=\left[\begin{array}{ccccc:cccc:c}-a & 0 & \cdots & 0 & 0 & 0 & 0 & \cdots & 0 & -2 A_{d} \\ \hdashline 1 & 0 & \cdots & 0 & 0 & 0 & 0 & \cdots & 0 & 0 \\ 0 & 1 & \ddots & \vdots & \vdots & 0 & \ddots & \ddots & \vdots & \vdots \\ \vdots & \ddots & \ddots & 0 & 0 & \vdots & \ddots & \ddots & 0 & 0 \\ 0 & \cdots & 0 & 1 & 0 & 0 & \cdots & 0 & 0 & 0 \\ \hdashline 1 & 0 & \cdots & 0 & 0 & \beta_{1} & 0 & \cdots & 0 & 0 \\ 1 & 0 & \ddots & \vdots & \vdots & 0 & \beta_{2} & \ddots & \vdots & \vdots \\ \vdots & \vdots & \ddots & 0 & 0 & \vdots & \ddots & \ddots & 0 & 0 \\ 1 & 0 & \cdots & 0 & 0 & 0 & \cdots & 0 & \beta_{l} & 0 \\ \hdashline 0 & 0 & \cdots & 0 & 0 & 0 & \cdots & 0 & 0 & -2 / T\end{array}\right]_{(m+l+2) \times(m+l+2)}$

$B_{1}=\left[\begin{array}{llll}A_{d} & 0 & \cdots & 0 \\ A_{d} & 0 & \cdots & 0\end{array}\right]_{2 \times(m+l+2)}^{T}, B_{2}=\left[\begin{array}{lllll}A_{d} & 0 & \cdots & 0 & 2 / T\end{array}\right]_{1 \times(m+l+2)}^{T}$,

$D_{12}=1$.

The resulting closed-loop system from $\bar{W}_{d}(s)$ to $Z(s)$ is thus as follows.

$$
T_{z \bar{w}_{d}}\left\{\begin{array}{l}
s \bar{\xi}(s)=\left(A+B_{2} F_{s}\right) \bar{\xi}(s)+B_{1} \bar{W}_{d}(s) \\
Z(s)=F_{s} \bar{\xi}(s)
\end{array}\right.
$$

Since $D(s)$ is an all-pass transfer function, for $\mathrm{H}_{2}$ minimization from $W_{n}(s)$ to $Z(s)$, equivalently from $W_{d_{1}}(s)$ to $Z(s)$, the system matrix of the closed-loop map is given by

$$
\left[\begin{array}{cc}
A+B_{2} F_{s} & B_{1} R_{1} \\
F_{s} & 0
\end{array}\right]
$$

where $R_{1}=\left[\begin{array}{ll}1 & 0\end{array}\right]^{T}$. On the other hand, for $H_{\infty}$ minimization from $W_{\theta}(s)$ to $Z(s)$, equivalently from $W_{d_{2}}(s)$ to $Z(s)$, the system matrix of the closed-loop map is given by

$$
\left[\begin{array}{cc}
A+B_{2} F_{s} & B_{1} R_{2} \\
F_{s} & 0
\end{array}\right]
$$

where $R_{2}=\left[\begin{array}{ll}0 & 1\end{array}\right]^{T}$.

\section{B. LMI design of the general form loop filters}

In order to meet the multiple design objectives mentioned in Section II, the following multi-objective control problem is considered, where $\lambda_{2}$ and $\lambda_{\infty}$ are weights for the trade-off of the two design objectives: noise bandwidth and gain/phase margins.

$$
\text { Minimize } \lambda_{2} \cdot v^{2}+\lambda_{\infty} \cdot \gamma
$$

over $N \in \mathfrak{R}^{1 \times(m+l+2)}, M=M^{T} \in \mathfrak{R}^{(m+l+2) \times(m+l+2)}, Q \in \mathfrak{R}$, $v \in \mathfrak{R}$, and $\gamma \in \mathfrak{R}$ satisfying:

$$
\left\{\begin{array}{cc}
H e\left(A M+B_{2} N\right) & B_{1} R_{1} \\
\left(B_{1} R_{1}\right)^{T} & -I
\end{array}\right)<0, \quad\left(\begin{array}{cc}
M & N^{T} \\
N & Q
\end{array}\right)>0,
$$

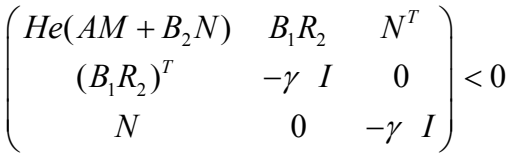

$$
\begin{aligned}
& \left(\begin{array}{cc}
\sin (\theta) \cdot H e\left(A M+B_{2} N\right) & \cos (\theta) \cdot \operatorname{Sh}\left(A M+B_{2} N\right) \\
\left(\cos (\theta) \cdot \operatorname{Sh}\left(A M+B_{2} N\right)\right)^{T} & \sin (\theta) \cdot H e\left(A M+B_{2} N\right)
\end{array}\right)<0 \\
& \left\{\begin{array}{r}
-2 h_{1} M-H e\left(A M+B_{2} N\right)<0 \\
2 h_{2} M+H e\left(A M+B_{2} N\right)<0
\end{array}\right.
\end{aligned}
$$

where the notation $\operatorname{He}(A)=A+A^{T}, \operatorname{Sh}(A)=A-A^{T}$ are used, $\mathfrak{R}$ denotes the set of all real numbers, and $\mathfrak{R}^{p \times m}$ denotes the set of all $p \times m$ matrices with all the entries in $\Re$. Denote the optimal solution by $\left(N_{\text {opt }}, M_{\text {opt }}, Q_{o p t}, v_{\text {opt }}\right.$, $\left.\gamma_{\text {opt }}\right)$.

Theorem 1: Given the time delay $T$, nonnegative integers $l, m$, the real values $a, \beta_{1}, \beta_{2}, \cdots \beta_{l}$, the weights $\lambda_{2}, \lambda_{\infty}$, the positive values $\theta$, and $h_{1}, h_{2}$ with $h_{2}<h_{1}$. If the optimization problem (6) is solvable, there exists a loop filter of the form

$$
F(s)=\frac{s}{s+a} \cdot \frac{1+(T / 2) \cdot s}{\left(1-f_{m+l+1}\right)+(T / 2) \cdot s} \cdot\left[\sum_{i=0}^{m} \frac{f_{i}}{s^{i}}+\sum_{i=m+1}^{m+l} \frac{f_{i}}{s-\beta_{i-m}}\right]
$$

with $F_{s}=\left[f_{0}, \cdots, f_{m+l+1}\right]=N_{\text {opt }} M_{\text {opt }}^{-1}$, such that

(i) the closed-loop system is stable,

(ii) $B_{n}<\frac{v_{o p t}^{2}}{2}, \mathrm{GM}>20 \log \frac{\gamma_{o p t}+1}{\gamma_{o p t}} \mathrm{~dB}, \mathrm{PM}>2 \sin ^{-1} \frac{1}{2 \gamma_{o p t}} \mathrm{deg}$,

(iii) the closed-loop poles lie within the intersection of the strip $\left(-h_{1},-h_{2}\right)$ and the conic sector with parameter $\theta$.

Proof: The assertions can be proved by applying Lemmas $1,2,3,4$ and the standard state feedback synthesis technique from [17], and the formulas (1) and (2).

Remark: The proposed design method provides an advantage for the designer to assign the filter poles a priori except that at $2\left(f_{m+l+1}-1\right) / T$. Moreover, the method is applicable to yield a filter of arbitrary order. Besides, the tracking capability of PLL loops can be enforced by increasing the number of the pure integrators in the filter.

An algorithm based on carrying out the optimization problem (6) and adjustment of the parameters $h_{1}, h_{2}$ and $\theta$ is 
presented as follows.

Algorithm 1: Given the desired noise bandwidth $B_{n}^{*}(\mathrm{~Hz})$, stability margins, i.e., GM/PM (equivalently $\left\|T_{\hat{w}_{\theta} w_{\theta}}\right\|_{\infty}=\gamma^{*}$ ), both of which are assumed to be greater than the minimal values in the individual case. Consider loop filer of form (3). Without loss of generality, $\lambda_{2}$ may be assumed to be unity.

Step 1 Select the weight $\lambda_{\infty}$, the values $h_{1}, h_{2}$ and $\theta$. Perform (6) to get the values of $B_{n}$ and $\gamma$.

Step 2 If $B_{n}>B_{n}{ }^{*}$, decreases $h_{2}$ and / or $h_{1}$. If $\gamma>\gamma^{*}$, decreases $h_{2}$ and/or increases $h_{1}$. Continue this process until $B_{n} \leq B_{n}^{*}$ and $\gamma \leq \gamma^{*}$.

Step 3 If the transient response is satisfactory, the filter design is complete; otherwise continue the following steps:

Step 3.1 If it is not good enough for overshoot, we decrease $\theta$ and perform (6) to get $B_{n}$ and $\gamma$. Go to Step 2.

Step 3.2 If it is not good enough for settling time, we increase $h_{2}$ and/or $\theta$ and perform (6) to get $B_{n}$ and $\gamma$. Go to Step 2 .

\section{PI Form LOOP FILTER DESIGN}

In this section, a new design of PI form loop filters is proposed for PLLs. The synthesis problem is formulated as a mixed $\mathrm{H}_{2} / \mathrm{H}_{\infty}$ control problem. A special coordinate transformation technique [21] is employed to deal with the problem.

\section{A. Problem reformulation}

In view of the formula (3) for the loop filer, if $l=0$ and both of the coefficients $a$ and $f_{m+1}$ are set to be zero, then (3) reduces to a PI form filter of order $m$. Specifically, define the state vector as

$$
X(s):=\left[Y(s), \frac{1}{s} Y(s), \cdots, \frac{1}{s^{m}} Y(s), \frac{1}{1+T / 2 \cdot s} \hat{U}(s)\right]^{T}
$$

With similar argument as part A of Section III, it is easy to verify that the resulting closed-loop system from $\bar{W}_{d}(s)$ to $Z(s)$ is as follows.

$$
T_{z \bar{w}_{d}}\left\{\begin{array}{l}
s X(s)=\left(A+B_{2} F_{0} C_{2}\right) X(s)+B_{1} \bar{W}_{d}(s) \\
Z(s)=F_{0} C_{2} X(s)
\end{array}\right.
$$

where

$$
A=\left[\begin{array}{ccccc:c}
0 & 0 & \cdots & 0 & 0 & -2 A_{d} \\
\hdashline 1 & 0 & \cdots & 0 & 0 & 0 \\
0 & 1 & \ddots & \vdots & \vdots & \vdots \\
\vdots & \ddots & \ddots & 0 & 0 & 0 \\
0 & \cdots & 0 & 1 & 0 & 0 \\
\hdashline 0 & 0 & \cdots & 0 & 0 & -2 / T
\end{array}\right]_{(m+2) \times(m+2)}
$$

$B_{1}=\left[\begin{array}{llll}A_{d} & 0 & \cdots & 0 \\ A_{d} & 0 & \cdots & 0\end{array}\right]_{2 \times(m+2)}^{T}, B_{2}=\left[\begin{array}{lllll}A_{d} & 0 & \cdots & 0 & 2 / T\end{array}\right]_{1 \times(m+2)}^{T}$,

$C_{2}=\left[\begin{array}{ll}I_{m+1} & 0_{(m+1) \times 1}\end{array}\right]$, and $F_{0}=\left[f_{0}, \cdots, f_{m}\right]$ is to be designed.

Again, for $H_{2}$ minimization from $W_{n}(s)$ to $Z(s)$ and for $H_{\infty}$ minimization from $W_{\theta}(s)$ to $Z(s)$ using $m$ th order PI form filter, it is required to solve (7) and (8) below, respectively, for the variables $Q_{2}, V, Q_{\infty}$ and $F_{0}$.

$\left\{\begin{array}{l}\left(\begin{array}{cc}H e\left\{A Q_{2}+B_{2} F_{0} C_{2} Q_{2}\right\} & B_{1} R_{1} \\ \left(B_{1} R_{1}\right)^{T} & -1\end{array}\right)<0, \\ \left.\begin{array}{cc}Q_{2} & \left(F_{0} C_{2} Q_{2}\right)^{T} \\ F_{0} C_{2} Q_{2} & V\end{array}\right)>0, \\ \operatorname{Tr}(V)<v^{2} .\end{array}\right.$

$$
\left\{\begin{array}{c}
\left(\begin{array}{ccc}
H e\left\{A Q_{\infty}+B_{2} F_{0} C_{2} Q_{\infty}\right\} & B_{1} R_{2} & \left(F_{0} C_{2} Q_{\infty}\right)^{T} \\
\left(B_{1} R_{2}\right)^{T} & -\gamma I & 0 \\
F_{0} C_{2} Q_{\infty} & 0 & -\gamma I
\end{array}\right)<0, \\
Q_{\infty}>0
\end{array}\right.
$$

Unfortunately, neither (7) nor (8) is jointly convex in the variables of its own. In the next subsection, we introduce a newly developed coordinate transformation technique [21] which will be used to deal with this difficult problem.

\section{B. Coordinate transformation technique}

Given $A \in \Re^{(m+2) \times(m+2)}, \quad B_{1} \in \Re^{(m+2) \times 2}, \quad B_{2} \in \Re^{(m+2) \times 1}$ and $C_{2}=\left[\begin{array}{ll}I_{m+1} & 0_{(m+1) \times 1}\end{array}\right]$. Define a set $\boldsymbol{S}$ of real matrices as follows:

$$
\boldsymbol{S}:=\left\{\left(\begin{array}{cc}
\boldsymbol{I}_{\boldsymbol{m + 1}} & 0_{(\boldsymbol{m}+1) \times 1} \\
\boldsymbol{T}_{21} & \boldsymbol{T}_{22}
\end{array}\right) \in \mathfrak{R}^{(\boldsymbol{m}+2) \times(\boldsymbol{m}+2)}: \boldsymbol{T}_{21} \in \mathfrak{R}^{1 \times(\boldsymbol{m}+1)}, \boldsymbol{T}_{22} \neq 0\right\} .
$$

It is readily checked that any matrix $T \in \boldsymbol{S}$ satisfies the property: $C_{2} T=C_{2}$. We will make use of the following two lemmas to transform the $H_{2}$ and $H_{\infty}$ minimization problems mentioned above to be LMI problems in certain coordinates, respectively.

Lemma 5 ( $\boldsymbol{H}_{2}$ performance) [21]: Given $\boldsymbol{v}>0$. The following statements are equivalent.

(i) there exist matrices $Q_{2}=Q_{2}^{T} \in \Re^{(m+2) \times(m+2)}, V \in \Re$ and $F_{0} \in \mathfrak{R}^{1 \times(m+1)}$ such that (7) is satisfied.

(ii) there exist matrices $N \in \mathfrak{R}^{1 \times(m+1)}, M \in \mathfrak{R}^{(m+1) \times(m+1)}, U_{2} \in \mathfrak{R}$, 
$V \in \mathfrak{R}$ and $T_{2} \in \boldsymbol{S}$ such that

$$
\begin{aligned}
& \left(H e\left\{\begin{array}{cc}
\left.\overline{\mathbf{A}}\left[\begin{array}{cc}
M & 0 \\
0 & U_{2}
\end{array}\right]+\overline{\mathbf{B}}_{\mathbf{2}} N \overline{\mathbf{C}}_{\mathbf{2}}\right\} & \overline{\mathbf{B}}_{\mathbf{1}} \\
\left(\overline{\mathbf{B}}_{\mathbf{1}}\right)^{T} & -I
\end{array}\right)<0,\right. \\
& \left(\begin{array}{cc}
{\left[\begin{array}{cc}
M & 0 \\
0 & U_{2}
\end{array}\right]} & \left(N \overline{\mathbf{C}}_{\mathbf{2}}\right)^{T} \\
N \overline{\mathbf{C}}_{\mathbf{2}} & V
\end{array}\right)>0, \quad \operatorname{Tr}(V)<v^{2} . \\
& \text { where } \\
& \overline{\mathbf{A}}=T_{2}^{-1} A T_{2}, \quad \overline{\mathbf{B}}_{\mathbf{1}}=T_{2}^{-1} B_{1} R_{1}, \overline{\mathbf{B}}_{\mathbf{2}}=T_{2}^{-1} B_{2}, \overline{\mathbf{C}}_{2}=C_{2} T_{2} .
\end{aligned}
$$

Lemma 6 ( $\boldsymbol{H}_{\infty}$ performance) [21]: Given $\gamma>0$. The following statements are equivalent.

(i) there exist matrices $Q_{\infty}=Q_{\infty}^{T} \in \mathfrak{R}^{(m+2) \times(m+2)}$ and $F_{0} \in \mathfrak{R}^{1 \times(m+1)}$ such that (8) is satisfied.

(ii) there exist matrices $N \in \mathfrak{R}^{1 \times(m+1)}, M \in \mathfrak{R}^{(m+1) \times(m+1)}, U_{\infty} \in \mathfrak{R}$ and $T_{\infty} \in \boldsymbol{S}$ such that

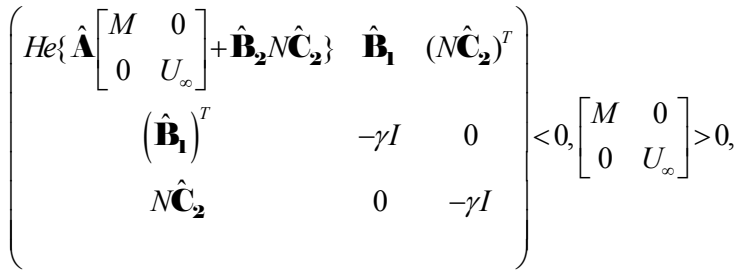

where

$$
\hat{\mathbf{A}}=T_{\infty}^{-1} A T_{\infty}, \quad \hat{\mathbf{B}}_{\mathbf{1}}=T_{\infty}^{-1} B_{1} R_{2}, \hat{\mathbf{B}}_{\mathbf{2}}=T_{\infty}^{-1} B_{2}, \hat{\mathbf{C}}_{\mathbf{2}}=C_{2} T_{\infty} .
$$

Note that the coordinate transformation matrices $T_{i}, i=2, \infty$, mentioned in statement (ii) of Lemma 5 and Lemma 6 can be set as follows.

$$
T_{i}=\left(\begin{array}{cc}
I_{m+1} & 0 \\
\left(\left(Q_{i, 11}\right)^{-1} Q_{i, 12}\right)^{T} & 1
\end{array}\right)
$$

where $Q_{i}=\left[\begin{array}{ll}Q_{i, 11} & Q_{i, 12} \\ Q_{i, 12}^{T} & Q_{i, 22}\end{array}\right], i=2, \infty$, were obtained from solving (7) and (8), respectively.

\section{LMI design of the PI form loop filters}

The problem of PI form loop filter design is formulated as a mixed $H_{2} / H_{\infty}$ optimization problem described as follows:

$$
\text { Minimize } \lambda_{2} \cdot v^{2}+\lambda_{\infty} \cdot \gamma
$$

over $F_{0} \in \mathfrak{R}^{1 \times(m+1)}, Q_{2}=Q_{2}^{T} \in \Re^{(m+2) \times(m+2)}, Q_{\infty}=Q_{\infty}{ }^{T} \in \mathfrak{R}^{(m+2) \times(m+2)}, V \in \Re$, $v \in \mathfrak{R}$, and $\gamma \in \mathfrak{R}$ satisfying (7) and (8)

Applying the coordinate transformation technique equivalently transforms the mixed $H_{2} / H_{\infty}$ optimization problem into the following optimization problem:

$$
\text { Minimize } \lambda_{2} \cdot v^{2}+\lambda_{\infty} \cdot \gamma
$$

over $N \in \mathfrak{R}^{1 \times(m+1)}, M \in \mathfrak{R}^{(m+1) \times(m+1)}, U_{2} \in \mathfrak{R}, U_{\infty} \in \mathfrak{R}, V \in \mathfrak{R}, \nu \in \mathfrak{R}$, and $\gamma \in \mathfrak{R}$ satisfying: (9) and (10).
Denote the locally optimal solution by $\left(\tilde{U}_{2}, \tilde{U}_{\infty}, \tilde{N}, \tilde{M}, \tilde{V}, \tilde{v}, \tilde{\gamma}\right)$

Theorem 2: Suppose $m>0$, and the weights $\lambda_{2}$ and $\lambda_{\infty}$ are given. If the optimization problem (12) is solvable, there exists a PI form loop filter $F(s)$ expressed as

$$
F(s)=\sum_{i=0}^{m} \frac{f_{i}}{s^{i}}
$$

with $F_{0}=\left[f_{0}, \cdots, f_{m}\right]=\tilde{N} \tilde{M}^{-1}$, such that

(i) the closed-loop system is stable,

(ii) $B_{n}<\frac{\tilde{v}^{2}}{2}, \mathrm{GM}>20 \log \frac{\tilde{\gamma}+1}{\tilde{\gamma}} \mathrm{dB}, \mathrm{PM}>2 \sin ^{-1} \frac{1}{2 \tilde{\gamma}} \mathrm{deg}$.

Proof: The assertions can be proved by applying Lemmas 5 and 6 and the formulas (1) and (2).

An algorithm based on carrying out the optimization problem (12) is presented as follows.

Algorithm 2: Given the desired noise bandwidth $B_{n}{ }^{*}(\mathrm{~Hz})$, stability margins (equivalently, $\left\|T_{\hat{w}_{\theta} w_{\theta}}\right\|_{\infty}=\gamma^{*}$ ) and the order $m$ of the loop filer. The weight $\lambda_{2}$ is selected to unity.

Step 1 Select initial feedback gain $F_{0}$.

Step 2 Solve (7) for minimizing $v$ to get $Q_{2}$, and solve (8) for minimizing $\gamma$ to get $Q_{\infty}$.

Step 3 Compute $T_{i}, i=2, \infty$, by (11).

Step 4 Compute $\overline{\mathbf{A}}, \overline{\mathbf{B}}_{\mathbf{1}}, \overline{\mathbf{B}}_{\mathbf{2}}, \overline{\mathbf{C}}_{\mathbf{2}}, \hat{\mathbf{A}}, \hat{\mathbf{B}}_{\mathbf{1}}, \hat{\mathbf{B}}_{\mathbf{2}}, \hat{\mathbf{C}}_{\mathbf{2}}$.

Step 5 Given $\lambda_{\infty}$. Perform (12) to get the values $B_{n}$ and $\gamma$. If $B_{n}>B_{n}{ }^{*}$, decreases $\lambda_{\infty}$. If $\gamma>\gamma^{*}$ increases $\lambda_{\infty}$. Continue this process until $B_{n} \leq B_{n}^{*}$ and $\gamma \leq \gamma^{*}$.

\section{Simulation Results}

In practical situation, an appropriate noise bandwidth of PLL in GPS receiver is selected to be around $15 \mathrm{~Hz}$ [25], i.e. $B_{n}^{*} \approx 15 \mathrm{~Hz}$. This design objective is enforced for all the cases discussed here. Without loss of generality, the phase detector gain $A_{d}$ is assumed to be unity. Furthermore, we assume that the loop delay is $10 \mathrm{~ms}$ and the standard deviation of the input Gaussian noise $n(t)$ is 0.05 . Two kinds of loop filter are designed by the proposed methods. The general form filters considered here are expressed by:

$$
F(s)=\frac{s+\frac{2}{T}}{s+\left(1-f_{m+1}\right) \cdot \frac{2}{T}} \cdot \sum_{i=0}^{m} \frac{f_{i}}{s^{i}} .
$$

Note that the class of PI form filters can be deemed as a special case of the general form filters because a general form filter reduces to be PI form when the parameter $f_{m+1}$ is set to be zero. The two types of filters are computed via Algorithm 1 and Algorithm 2. 
TABLE 1 (A) and (B) show the transfer functions of the two types of loop filters and their design parameters. In TABLE 1 (B), the value inside each bracket denotes the number of iteration. For example, the symbol $F_{0}(0)$ denotes the feedback gain initially chosen. Several performance indices are evaluated for the resulting PLLs as shown in TABLE 2. The gain and phase margins on the list are the guaranteed values computed by the formulas (1) and (2), which are approximately the same for both designs. For overshoot performance index, the resulting PLLs yield almost the same quantity. Compared with PI form filters, the general form filters have slightly smaller settling time.

TABLE 1 (A) GENERAL FORM LOOP FILTERS

\begin{tabular}{|c|l|c|}
\hline \# integrator & Design parameters & Loop filter $F(s)$ \\
\hline \multirow{2}{*}{1} & $\begin{array}{l}\lambda_{2}=1, \lambda_{\infty}=1 \\
\theta=\pi / 11.64, h_{1}=210, h_{2}=12\end{array}$ & $\frac{(46.3 \mathrm{~s}+392.4) \cdot(s+200)}{s(s+288.68)}$ \\
\hline \multirow{2}{*}{2} & $\lambda_{2}=1, \lambda_{\infty}=15$ & $\frac{\left(46.85 s^{2}+326.18 \mathrm{~s}+628.16\right) \cdot(\mathrm{s}+200)}{s^{2}(s+282.44)}$ \\
\hline \multirow{2}{*}{3} & $\theta=\pi / 40, h_{1}=190, h_{2}=3.48$ & $\frac{\left(38.5 s^{3}+303.95 s^{2}+828.97 s+733.66\right) \cdot(s+200)}{s^{3}(s+235.08)}$ \\
\hline
\end{tabular}

\begin{tabular}{|c|c|c|}
\hline \# integrator & Design parameters & Loop filter $F(s)$ \\
\hline 1 & $\begin{aligned} F_{0}(0) & =\left[\begin{array}{ll}50 & 510\end{array}\right], \\
\lambda_{2}(0) & =1, \lambda_{\infty}(0)=362, \\
F_{0}(1) & =\left[\begin{array}{ll}33.36 & 267.94\end{array}\right]\end{aligned}$ & $33.36+\frac{267.94}{s}$ \\
\hline 2 & $\begin{array}{l}F_{0}(0)=\left[\begin{array}{lll}100 & 1000 & 3000\end{array}\right] \\
\lambda_{2}(0)=1, \lambda_{\infty}(0)=330 \\
F_{0}(1)=\left[\begin{array}{lll}34.44 & 226.19 & 497.08\end{array}\right]\end{array}$ & $34.44+\frac{226.19}{\mathrm{~s}}+\frac{497.08}{s^{2}}$ \\
\hline 3 & $\begin{array}{l}F_{0}(0)=\left[\begin{array}{llll}100 & 1000 & 1970 & 2970\end{array}\right] \\
\lambda_{2}(0)=1, \lambda_{s}(0)=284 \\
F_{0}(1)=\left[\begin{array}{llll}33.20 & 266.16 & 517.88 & 742.60\end{array}\right]\end{array}$ & $33.2+\frac{266.16}{\mathrm{~s}}+\frac{517.88}{s^{2}}+\frac{742.6}{s^{3}}$ \\
\hline
\end{tabular}

\begin{tabular}{|c|c|c|c|c|c|}
\multicolumn{6}{c|}{ TABLE 2 The performance indices for noise bandwidth $15 \mathrm{~Hz}$} \\
\hline $\begin{array}{c}\text { Filter } \\
\text { type }\end{array}$ & $\begin{array}{c}\# \\
\text { integrator }\end{array}$ & $\begin{array}{c}\text { Rise } \\
\text { time } \\
(\mathrm{sec})\end{array}$ & $\begin{array}{c}\text { Overshoot } \\
(\%)\end{array}$ & $\begin{array}{c}\text { Settling } \\
\text { time } \\
(\mathrm{sec})\end{array}$ & $\begin{array}{c}H_{\infty} \\
\text { norm }\end{array}$ \\
\hline \multirow{2}{*}{$\begin{array}{c}\text { General } \\
\text { form }\end{array}$} & 1 & 0.03 & 17.8 & 0.306 & 1.21 \\
\cline { 2 - 6 } & 2 & 0.03 & 16.8 & 0.379 & 1.2 \\
\hline \multirow{2}{*}{$\begin{array}{c}\text { PI } \\
\text { Form }\end{array}$} & 3 & 0.03 & 20 & 0.355 & 1.24 \\
\cline { 2 - 6 } & 1 & 0.028 & 18.1 & 0.311 & 1.21 \\
\cline { 2 - 6 } & 3 & 0.028 & 16.8 & 0.388 & 1.19 \\
\hline
\end{tabular}

\begin{tabular}{|c|c|c|c|}
\hline Filter type & \# integrator & Gain margin & Phase margin \\
\hline \multirow{3}{*}{ General form } & 1 & $5.23 \mathrm{~dB}$ & $48.81 \mathrm{deg}$ \\
\cline { 2 - 4 } & 2 & $5.26 \mathrm{~dB}$ & $49.25 \mathrm{deg}$ \\
\cline { 2 - 4 } & 3 & $5.14 \mathrm{~dB}$ & $47.56 \mathrm{deg}$ \\
\hline \multirow{3}{*}{ PI Form } & 1 & $5.23 \mathrm{~dB}$ & $48.81 \mathrm{deg}$ \\
\cline { 2 - 4 } & 2 & $5.26 \mathrm{~dB}$ & $49.69 \mathrm{deg}$ \\
\cline { 2 - 4 } & 3 & $5.14 \mathrm{~dB}$ & $47.56 \mathrm{deg}$ \\
\hline
\end{tabular}

Next we use the software MATLAB's SIMULINK Version 7.3 to simulate the resulting PLLs. A nonlinear system model, as shown in Fig. 6, is used, where the function of the phase detector is modeled as $A_{d} \sin (\bullet)$ instead of a constant gain $A_{d}$.

Figures 7, 8 and 9 show the frequency step responses of second-order, third-order and fourth-order PLLs, respectively. Overall, these show that the resulting PLLs using both general form filters and PI form filters can track the input trajectory swiftly when the phase ramp signal of 40 $\mathrm{rad} / \mathrm{sec}$ is applied. The transient responses of the resulting PLLs are similar.

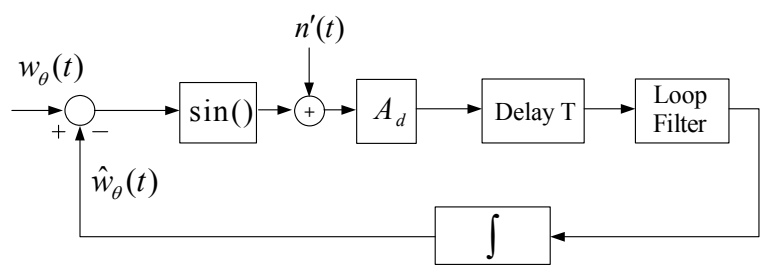

Fig. 6 The block diagram of PLL model
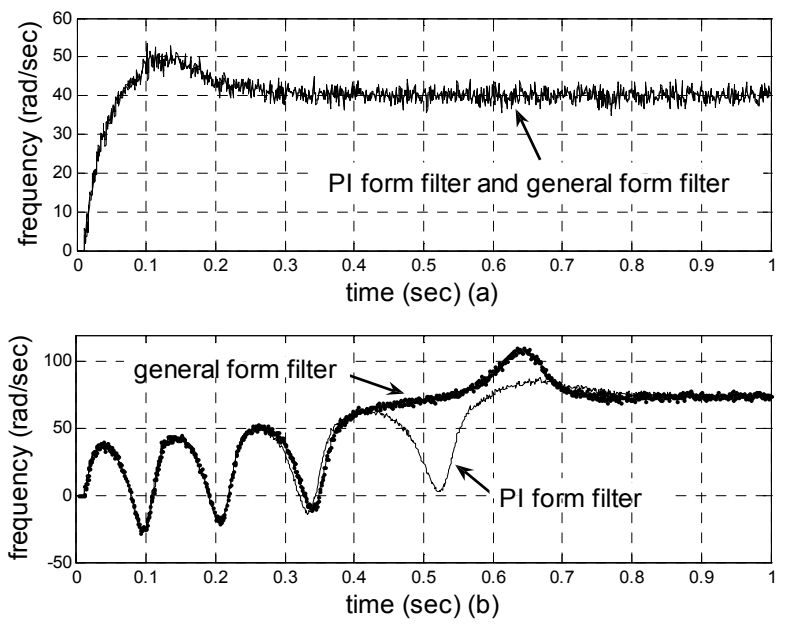

Fig. 7 The frequencies of VCO output when a frequency change (ramp input phase): (a) $40 \mathrm{rad} / \mathrm{sec}$ and (b) $75 \mathrm{rad} / \mathrm{sec}$ is applied to second-order PLLs.
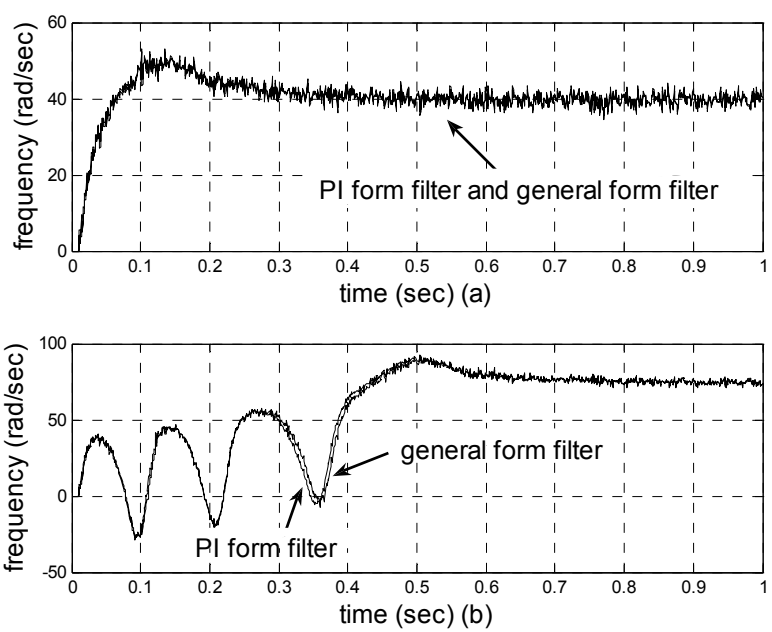

Fig. 8 The frequencies of VCO output when a frequency change (ramp input phase): (a) $40 \mathrm{rad} / \mathrm{sec}$ and (b) $75 \mathrm{rad} / \mathrm{sec}$ is applied to third-order PLLs.

The pull-out phenomenon occurs if a larger frequency offset signal of $75 \mathrm{rad} / \mathrm{sec}$ is encountered. Through a slow pull-in process, the loop can get locked again as long as it is within the lock-in range. It is observed that the PLLs with 
general form loop filters exhibit better tracking capability and shorter pull-in time than those with PI form filters. Particularly, when the PLLs suffer from a greater frequency offset, those with general form filters can provide an effective control to speed up the tracking capability. Nevertheless, the performances of the PI form filters are very close to those of the general form filters and are easier to be implemented.
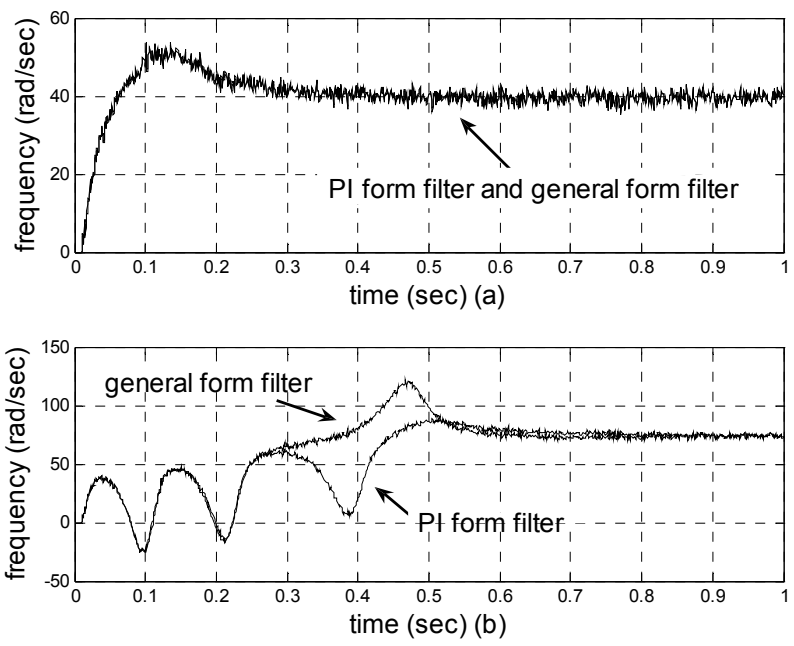

Fig. 9 The frequencies of VCO output when a frequency change (ramp input phase): (a) $40 \mathrm{rad} / \mathrm{sec}$ and (b) $75 \mathrm{rad} / \mathrm{sec}$ is applied to fourth-order PLLs.

\section{CONCLUSIONS}

We have presented two new loop filter designs for PLLs with undesired but inevitable loop delay. Both of the two methods considered to tradeoff the design objectives such as small noise bandwidth and large gain/phase margins via multi-objective control techniques. When first-order Padé approximation for the delay term is applied, unlike the results of direct application of the existing multi-objective control technique leading to filters of only second-order which may not be able to meet the increasingly tough performance requirements of PLLs, our proposed methods can yield filters with arbitrary order. Furthermore, almost all except one of the general form filter poles can be assigned a priori. A special coordinate transformation technique has been employed to deal with the design of the popular class of PI form filters. Algorithms have been provided for the proposed design methods. Simulation results demonstrate the good performance of the resulting PLLs.

\section{ACKNOWLEDGMENT}

This work was supported in part under Grants NSC 94-2213-E-002-079, NSC 94-2213-E-032-019 and NSC 90-2213-E-032-008.

\section{REFERENCES}

[1] A. Blanchard, Phase-Locked Loops: Application to Coherent Receiver Design. Wiley: New York, 1978.
[2] A. J. Viterbi, Principles of Coherent Communications. McGraw-Hill: New York, 1966.

[3] R. E. Best, Phase-locked loop: Design. Simulation, and Applications, McGraw-Hill International, 1997.

[4] F. M. Gardner, Phaselock Techniques. John Wiley, New York, NY, third ed., 2005.

[5] A. Abu-Rgheff, M.N. Sumartana, I.G.G, "Carrier phase tracking in digital radio communications," Electronics Letters, vol. 34, pp. $2306-2307,1998$

[6] A. De Gloria, D. Grosso, M. Olivieri, and G. Restani, “A novel stability analysis of a PLL for timing recovery in hard disk drives," IEEE Trans. Circuits and Systems-I: Fundamental Theory and Applications, vol. 46, no. 8, pp. 1026-1031, 1999.

[7] M. F. Lai, M. Nakano, and G.C. Hsieh, "Application of Fuzzy logic in the phase-locked loop speed control of induction motor drive," IEEE Trans. on Industrial Electronics, vol. 43, no.6, pp. 630-639, 1996.

[8] J. W. Ahn, S. G. Oh, S. Y. Pyo, C.U. Kim, and Y. M. Hwang, "Digital PLL technique for precise speed control of SR Drive, Power Electronics Specialists Conference (PESC99) 30th Annual IEEE, vol. 2,1999 , pp. $815-819$.

[9] G. C. Hsieh and J. C. Huang, Phase-Locked Loop Techniques- A Survey," IEEE Trans on Industrial Electronics, vol. 43, no.6, pp. 609-615, 1996.

[10] D. Y. Abramovitch, "Analysis and design of a third order phase-lock loop," in Proceeding of the IEEE Military Communications Conference, vol. 2, October 1988, pp. 455-459.

[11] D. Y. Abramovitch, "Lyapunov Redesign of analog phase-lock loops," IEEE Trans. on Communication, vol. 38, pp. 2197-2202, December 1990.

[12] O. Yaniv, D. Raphaeli, "Near-optimal PLL design for decision feedback carrier and timing recovery," IEEE Trans. on Communication, Vol. 49, pp. 1669 - 1678, Sept. 2001.

[13] M. Vidyasagar, Control System Synthesis-A Factorization Approach. MIT press, 1985.

[14] O. Yaniv, Quantitative Feedback Design of Linear and Nonlinear Control Systems. Norwell, MA: Kluwer, 1999.

[15] V. Suplin and U. Shaked, "Robust H-infinity control of phase-locked loops with polytopic type uncertainties," Int. J. Robust and Nonlinear Control, vol. 11, pp. 305-314, 2001.

[16] N. S. Nise, Control Systems Engineering. John Wiley \& Sons, Inc., $4^{\text {th }}$ edition, 2004

[17] S. Boyd, L. EL Ghaoui, E. Feron, and V. Balakrishnan, Linear Matrix Inequalities in System and Control Theory, SIAM, Philadelphia, 1994

[18] C. Scherer, P. Gahinet, and M. Chilali, "Multiobjective output feedback control via LMI optimization" IEEE Trans. Autom. Control, vol. 42, pp. 896-911, 1997

[19] M. Chilali and P. Gahinet, "Hळ design with pole placement constraints: an LMI approach," IEEE Trans. Autom. Control, vol. 41, pp. 358-367, 1996.

[20] P. Gahinet, A. Nemirovski, A. Laub, and M. Chilali, LMI Control Toolbox, The MathWorks Inc., Natick, MA, 1995.

[21] Y. C. Chu, Design of Reduced-order Controllers for Weighted Sensitivity Minimization Problem, MS thesis, Tamkang University, June 2006

[22] K. Zhou, and J. C. Doyle, Essentials of Robust Control, Prentice-Hall, Inc., New Jersey, 1998.

[23] C. E. de Souza and U. Shaked, "An LMI method for output-feedback $H_{\infty}$ control design for system with parameter uncertainty ", in Proc. IEEE Conf. Decision and Control, Tampa, Florida USA, pp.1777-1779, 1998.

[24] Y.S. Chou, W.L. Mao, Y.C. Chen and F.R. Chang, "A Novel Loop Filter Design for Phase-Locked Loops," IEEE International Conference on Systems, Man, and Cybernetics (IEEE SMC2006), Taipei, Taiwan, pp. 2932-2938, Oct. 2006.

[25] M. S. Braasch and A. J. Van Dierendonck, "GPS receiver architectures and measurements," in Proceedings of the IEEE, vol. 87, pp. 48-64, 1999. 